ce point aux pages éclairantes rédigées pour le tome trois de l'Histoire de la France parue au Seuil ${ }^{2}$. De la même façon, on aurait aimé que prît davantage d'ampleur l'analyse du moment décisif de 1791 où se cristallisa la rupture entre deux logiques, celle, politique, de la Nation, et celle d'un milieu qui ne parvint jamais dès lors à se penser comme une addition d'individualités ni à assumer pleinement sa dimension collective.

De cette lecture, rendue difficile par cela même qui la rend attrayante, l'extraordinaire foisonnement d'exemples minutieusement disséqués, autant d'îlots où le lecteur est exposé à une déviance métonymique, on retire la certitude qu'un certain nombre d'obstacles sont définitivement levés, et que le problème de la relation État-entrepreneurs est désormais posé en termes adéquats et féconds. Il n'en rend que plus aiguë l'interrogation sur ce qu'est, finalement, le libéralisme. Pur effet de discours? Ou dialogue pour faire coïncider une construction politique avec des configurations, des pratiques, des représentations qui lui échappent? En même temps que les " Deux rêves du Commerce ", Jean-Pierre Hirsch nous fait approcher les deux rêves de l'historien : donner à voir une société ou une époque "dans la familiarité essentielle à la compréhension des problèmes sociaux", et donner à penser des problèmes qui sont plus que jamais d'actualité : la pertinence des réponses libérales et du discours de la concurrence aux défis des contradictions et des inégalités sociales comme des exigences de la croissance.

Dominique Margairaz.

\title{
HISTOIRE DU SAVOIR ÉCONOMIQUE
}

Ars Mercatoria. Eine analytische Bibliographie. Bd $1: 1470-1600$. Mit einer Einleitung in deutscher und französischer Sprache. Herausgegeben von Jochen Hoock und Pierre Jeannin. 6 Bände. Paderborn, Schöningh, 1991. 17,5 x 24,5, LIV-432 p.

La connaissance du monde des marchands est enrichie de la publication du premier tome des Ars Mercatoria, répertoire bibliographique des manuels et traités à l'usage des marchands, qui recense les ouvrages dont la première parution attestée est antérieure à 1601 , en attendant la livraison ultérieure d'un volume qui couvrira les années 1601-1820. Près de deux mille références, plus de milke auteurs repérés dans toute l'aire de civilisation européenne en dépit des conditions inégales d'investigation, signalés par un descriptif précis (auteur, titre intégral, éditeur, imprimeur, lieu de consultation ou source secondaire où se trouve mentionné l'ouvrage, codage analytique, etc.). Un index par auteurs, une chrono-

2. André Burguière, Jacques Revel, dir., Histoire de la France. 3. Les Conflits, Paris, Seuil, 1990. 
logie faisant état des rééditions ou émissions nouvelles offrent aux chercheurs des possibilités d'entrées multiples. Le répertoire alphabétique par auteur, suivi des anonymes, est assorti d'une vingtaine de pages de tableaux et graphiques (en allemand) qui constituent une première approche statistique de l'évolution des parutions entre 1470 et 1599 , selon les lieux ou les thèmes principaux, suggérant une géographie différentielle de la production du savoir marchand.

L'introduction (bilingue), sous la plume des deux responsables de l'enquête, précise les conditions et les difficultés d'élaboration d'un tel corpus. Savoir en construction dont les frontières sont forcément incertaines, le savoir marchand est un savoir mouvant dont les contours et les articulations varient non seulement en fonction des exigences de la demande, mais au gré de l'évolution des disciplines qui le soutiennent et l'irriguent : mathématiques, droit, géographie, que la pratique du négoce tend à son tour à stimuler ${ }^{3}$. La question de la délimitation du corpus, c'est-à-dire de la pertinence des choix opérés est donc au centre de ces lignes qui contribuent à éclairer la logique et les arbitrages inévitables, qui ont préludé à la création de la base de données, et à fonder sa scientificité. En dehors d'un projet pédagogique clairement énoncé, les «manuels " n'appartiennent pas à un genre homogène. Leur définition, comme instrument pratique servant à l'apprentissage ou à l'exercice du métier de marchand, renvoie à des contenus hétérogènes qui vont de l'information sur les changes et les monnaies à la pratique du calcul et de la comptabilité, de la description géographique au commentaire des règles de droit, des techniques marchandes aux "usages " largement entendus, et dont la connaissance est indispensable tant pour la réalisation de profits que pour l'élargissement du crédit. L'élaboration de catégories analytiques n'en est que plus. ardue et la prétention à l'exhaustivité inscrite dans les bornes des choix initiaux.

Au demeurant, l'objet de l'enquête dépasse l'horizon de la compilation bibliographique, puisqu'il s'agit de mettre en lumière « la constitution, la transmission, la transformation des savoirs et des savoir-faire des marchands depuis la fin du Moyen Âge jusqu'à l'ère industrielle ", dans une perspective comparative. Dans un article récent, P. Jeannin s'est expliqué sur le statut de ces manuels ${ }^{4}$, aidemémoire plus qu'outil d'apprentissage à proprement parler, véhicule d'un savoir circulant plus que support d'innovation. Instruments pratiques destinés à figurer sur le comptoir du négociant, ils n'appartiennent pas à la sphère des ouvrages savants sagement rangés sur les rayons de bibliothèques. Interrogés de façon appropriée, ils témoignent donc bel et bien des pratiques, des problèmes, de l'outillage mental requis pour l'exercice de la profession, des décalages entre les différentes aires où les acteurs déployent leur activité. L'étude de cas menée par J. Hoock et J. Bottin dans le même ouvrage ${ }^{5}$ démontre elle aussi, s’il en était

3. Voir à cet égard la contribution de Paul BENofT, " Calcul, algèbre et marchandise ", in Eléments d'histoire des sciences, dir. Michel Serres, Paris, Bordas, 1989.

4. Pierte Jfannin, « Les manuels de pratique commerciale imprimés pour les marchands français (xvi"-xvı" siècle) ", in Le Négoce international, dir. François M. Crouzet, Paris, Economica, 1989 , p. 35-57.

5. Jacques Botrin, Jochen Hoock, " Structures et formes d'organisation du commerce à Rouen au début du xvil siècle : le cas de Michel Van Damme ", in op. cit. supra n. 2, p. 59-93. 
besoin, l'ampleur des perspectives ouvertes à la recherche par la publication d'un tel corpus, dont le succès n'a pu être assuré que grâce au concours de nombreuses collaborations auxquelles les deux auteurs rendent hommage.

Dominique Margairaz.

« L'invention de l'emploi », Économies et societés, t. XXIII, 10, 1989, Cahiers de l'I.S.M.E.A, série Economia, Histoire de la pensée économique, P.E., $\mathrm{n}^{\circ} 12$, 1989.

Le thème central de ce dossier, "l'invention de l'emploi ", rappelle le livre de R. Salais, B. Reynaud et N. Baverez : L'Invention du chomage (1986) qui étudie l'apparition et le développement d'une notion essentielle à la théorie et à l'histoire économiques du $\mathrm{xx}^{e}$ siècle. Dans son introduction, B. Gazier situe explicitement ce numéro d' $E$ conomia en prolongement de l'ouvrage de $\mathrm{R}$. Salais : en effet, il est amené à traiter autant đu chômage que de l'emploi tant les deux notions sont dépendantes l'une de l'autre. Soulignons cependant que seuls quelques aspects, très ponctuels, de ce vaste sujet sont abordés même si ce dossier a le mérite de multiplier les angles d'approche, de l'étude d'un auteur aux interrogations épistémologiques en passant par le problème historique de l'emploi féminin. Il en résulte inévitablement un certain manque de cohérence et une impression de dispersion qui exclut toute vision globale de l'invention de l'emploi. De cet ensemble de cinq études, retenons en particulier les deux articles de $\mathbf{M}$. Mansfield et C. Centi dont la première qualité est de traiter un aspect essentiel du sujet : le lien entre emploi et organisation du marché du travail, avec l'étude de deux économistes secondaires mais dignes d'intérêt : W. Beveridge et G. de Molinari.

L'ouvrage de Beveridge, Unemployment. A Problem of Industry (1909), est intéressant par son insistance sur la nécessité d'organiser l'emploi comme prélude à toute tentative pour traiter la question du chomage. C'est l'institution d'un emploi normalisé qui peut seule mettre en évidence un chómage économique conceptuellement distíngué des problèmes de pauvreté ou de sous-emploi chronique. $\AA$ une époque où les travailleurs occasionnels et le travail temporaire sont encore très nombreux dans les faits, Beveridge estime que cette séparation est nécessaire pour restaurer l'efficacité d'ensemble de la main-d'ceuvre et constituer une élite ouvrière à la fois mieux rémunérée et plus efficiente. C'est en ce sens que l'idée de chômage est utile car elle est le complément naturel de celle d'emploi. Puisque celui-ci doit résulter d'un marché du travail organisé qui permet une interaction entre une offre et une demande de travail, sa détermination rationnelle implique et suppose la création d'un chômage temporaire et variable - on dirait aujourd'hui frictionnel - capable de permettre cette confrontation. Dès lors, la mise en place d'un tel marché est essentiel car son fonctionnement permet d'identifier les " travailleurs honnêtes " désireux de trouver un emploi et qui doivent 
être aidés en cas de chômage. Les autres, ceux qui refusent de saisir les opportunités que propose le marché, ne doivent pas profiter de la mème sollicitude. Cette objectivation du concept d'unemployment grâce aux lois d'un marché organisé est pour Beveridge la condition à la fois d'une aide utile aux "bons travailleurs " et de la création d'une main-d'œuvre performante. Gustave de Molinari (1819-1912) a beaucoup écrit au cours de sa longue existence. Il défendra toujours le libreéchange, ce qui l'amène à développer des thèses originales sur l'emploi. Pour améliorer le sort des classes ouvrières, il faut que les offreurs de travail soient parfaitement mobiles, ce qui suppose la création de bourses du travail comme il existe des bourses des valeurs. Cette idée utopique de sa jeunesse, Molinari ne la reniera pas mais il la formalisera mieux grâce à l'idée de " mobilisabilité " du travail, c'est-à-dire de la prise en charge par l'ouvrier de la vente de ce demier, qui débouche sur l'agencement d'un marché général du travail. Outre une meilleure organisation de l'emploi, celui-ci permet une dépersonnalisation des rapports entre capital et travail, garantie de la paix sociale. Cette mobilisation ouvrière a pour conséquence une marchandisation du travail dont la rémunération résulte alors des lois économiques de l'échange qui, par leur objectivité et leur neutralité, sont de nature égalitaire. Elle a de plus des implications politiques car les mauvais régimes, où la liberté ne règne pas, seront fuis. Pour Molinari, comme pour bien des réformateurs du xix ${ }^{t}$ siècle, l'économie est donc profonđément politique car elle est d'abord une pratique sociale. Le concept de mobilisation est en ce sens bien différent de la simple mobilité puisqu'elle suppose pour ètre efficace que chaque salarié se comporte en bon gestionnaire de son capital personnel. Producteur de son propre travail, l'ouvrier libre doit savoir l'exploiter au mieux. Molinari apparaît par là comme un adepte precooe du capital humain. Son libéralisme a cependant peu à voir avec celui de Walras car il postule que la construction des sujets économiques se confond avec celle de l'économie alors que le maître de Lausanne écarte le problème de cette construction au profit de l'élaboration d'une science économique sans sujets puisque seuls les facteurs productifs interviennent.

Le rapprochement des deux analyses de Beveridge et Molinari que suggère ce numéro d'Économies et sociétés n'est pas sans intérêt. Il permet, en effet, d'opposer l'apparition de deux notions antagonistes de l'emploi. L'une repose sur l'existence d'un marché du travail qui doit être construit pour permettre un fonctionnement économique autonome, abstraction qui s'appuie paradoxalement sur des considérations empiriques (la distinction entre le chômage et le sous-emploi ou le travail temporaire). L'autre refuse, au contraire, l'idée de marché du travail au profit d'une approche sociale et politique de l'economique, en apparence donc plus réaliste, mais qui débouche sur un capitalisme utopique où la détermination de l'emploi résulte de l'action de sujets dont les comportements construits sont très éloignés des pratiques réelles. Comment ne pas relire aujourd'hui avec profit ces deux ceuvres à l'heure où la pertinence des analyses menées par les théories de lemploi, en particulier quant à la notion de marché du travail, est souvent remise en cause au nom précisément des contradictions qui opposent l'abstraction des hypothèses et la nécessaire prise en compte du réel ? 
Ingrid Kretschmer, Johannes Dörflenger, Franz WaWruk, Lexikon zur Geschichte der Kartographie von den Anfängen bis zum ersten Weltkrieg. $\mathrm{Bd} \mathrm{C} / 1$ et Bd C/2. Vienne, Franz Deuticke, 1986. $17 \times 24,5,652$ p., XVI Farbtafeln et 463-987 p., index (“ Enzyklopädie der Kartographie »).

Vaste entreprise que ce dictionnaire, fruit d'une coopération internationale. En deux volumes d'un millier de pages sur deux colonnes en petits caractères, et en près de six cents vedettes, il a l'ambition de couvrir toute l'histoire de la cartographie, depuis la Préhistoire jusqu'à la Première Guerre mondiale, sans oublier les mondes extérieurs à l'Europe. Environ cent cinquante savants y ont participé. On notera le petit nombre de contributeurs français, $c$ qui reflète, en premier lieu sans doute, la faible place qu'occupent chez nous les études d'histoire de la géographie, et aussi le parti des éditeurs, qui ont naturellement privilégié l'Europe centrale. Entreprise nécessaire aussi, en ce qu'elle fait le point des connaissances dans un domaine de recherche situé au carrefour de l'histoire des sciences et de l'histoire des représentations, où les synthèses de qualité ne sont pas légion.

De larges principes ont présidé au choix des vedettes, que l'on peut rassembler en cinq catégories : 1) techniques (fondements mathématiques et géodésiques, méthodes de représentation et de reproduction) ; 2) cartographes notables, historiens de la cartographie et, secondairement, institutions intéressées à la production de cartes ; 3) types de cartes ; 4) périodes et domaines de production cartographique (par exemple, "Renaissance", "cartographie britannique "); 5) histoire de la représentation đes États et régions. Aucun domaine n'a donc été laissé de côté.

D'un dictionnaire couvrant l'histoire d'une discipline, il est parfois à craindre qu'il déçoive les spécialistes de toutes les périodes, par un traitement trop cursif des données, et une appréciation correcte du contenu ne peut s'opérer pertinemment que pour un domaine où le critique a quelque compétence. Nous examinerons donc ce qu'il en est de la cartographie médiévale.

L'impression générale est plus que satisfaisante. Certes, on souhaiterait trouver plus d'une dizaine de monographies consacrées aux cartographes médiévaux. Mais les personnalités essentielles sont citées dans des articles d'ordre plus général. Le Moyen Âge bénéficie, d'autre part, d'un paragraphe dans les notices consacrées aux unités régionales ou aux types de cartes. Il est toutefois regrettable que ces développements se bornent souvent à l'énumération rapide de quelques cartes. Mais il arrive que les articles portant sur tel domaine de production cartographique analysent de façon détaillée les monuments médiévaux, comme l'excellent "Italien ». Enfin, la période est présentée par quelques entrées générales ("Christliche Kartographie ", "Katalanisch-Mallorcinische Kartographie ", "Klimazonen Karte », "Mittelalter », " Portolan », « TO-Karte ») qui fournissent d'utiles synthèses, ainsi que par des monographies sur des monuments célèbres.

Le spécialiste ne sera sans doute pas toujours d'accord avec toutes les interprétations. Mais la multiplicité des points de vue permettra de corriger utilement certaines affirmations. Ainsi, à propos du « Mittelalter », il est dit que la carto- 
graphie médiévale, science auxiliaire de la théologie déterminée tout entière par des motifs religieux, n'a pas d'utilité pratique, à l'exception des cartes nautiques. L'opinion serait pour le moins à nuancer, en s'entendant d'abord sur la notion de pratique. Mais l'auteur de l'article "Strassenkarte» affirme d'ailleurs que des cartes routières médiévales servirent aux pèlerins, aux marchands, aux militaires et aux courriers. Nul doute que la réflexion du lecteur ne soit stimulée par des contradictions de cette nature.

Ces réserves sont de peu d'importance. Néanmoins, on relèvera une absence, et certaines insuffisances. Le domaine de la cartographie à grande échelle (cartes de régions, de terroirs) n'est à peu près pas traité en tant que tel, pour la période médiévale, peut-être à cause du privilège accordé à l'espace centre-européen, où les témoins médiévaux semblent rarissimes. De même, l'article « Grenzkarte » ne comporte aucun exemple médiéval. Surtout, l'histoire des techniques souffre de quelques graves erreurs, peut-être dues au fait que les rédacteurs, non médiévistes, ne paraissent pas avoir toujours travaillé de première main. Dans l'article « Kompass ", on lit, entre autres, que l'Amalfitain Flavio Gioja fut responsable de l'amélioration de la boussole, et que la détermination de la position d'un navire grâce à l'échelle de Jacob et à la boussole commença dès le début du xiv siècle. Il s'agit de légendes depuis longtemps détruites. Contrairement à ce qui est affirmé dans l'article " Breitenbestimmung ", la détermination de la latitude, à terre, ne débuta pas au XIv" siècle en Allemagne du Sud, et la méthode n'en était pas empruntée aux Vikings. Il y avait beau temps que les astronomes universitaires, en France, en Italie, en Espagne et en Angleterre, mesuraient la hauteur du pôle à l'aide de l'astrolabe. Selon l'article « Portolane " (c'est-à-dire les livres d'instructions nautiques, ainsi que les cartes nautiques), généralement bien informé, la première attestation du terme est de 1285, avec renvoi à Du Cange. Mais l'auteur du Glossarium donnait comme référence à portulani un texte, en effet de 1285 , désignant sous ce terme des personnages « qui portubus maris uel fluminum praesunt ": rien à voir avec l'objet de l'article!

Il serait hors de propos pour un tel ouvrage de se livrer à une critique exhaustive. Dans leur majorité, les notices sont de bonne tenue, même si on les souhaiterait parfois plus fouillées (ainsi, il n'est pas question des représentations de Rome dans l'article "Stadtplan "). Une lecture croisée, aidée par de nombreux renvois, donnera rapidement une idée précise de n'importe quel domaine de l'histoire de la cartographie, que l'on pourra aisément approfondir grâce aux excellentes bibliographies qui accompagnent chaque article. L'illustration est abondante et judicieusement choisie.

Ce Lexikon offre une imposante contribution à l'histoire de la cartographie. Loin de faire double emploi avec d'autres encyclopédies, cet instrument de travail sera de consultation indispensable, pour le spécialiste et pour quiconque souhaitera prendre une vue rapide d'un sujet peu familier.

Patrick Gautier Dalche. 
Gilles Montigny, De la ville à l'urbanisation. Essai sur la genèse des études urbaines françaises en géographie, sociologie et statistique sociale. Préf. de Marcel RonCaYolo. Paris, L'Harmattan/Plan urbain, 1992. 16 x 24, 376 p., bibliogr., bibliogr. des cuvres de P. Meuriot, index ("Villes et entreprises ").

Raccourci d'une vaste thèse, cet essai porte, comme l'indique son sous-titre, sur " la genèse des études urbaines françaises en géographie, sociologie et statistique sociale ». Une genèse mal connue, voire oubliée de ceux qui s'intéressent à la ville aujourd'hui. L'auteur la fait coïncider avec la période 1890-1920, moment où naissent, c'est-à-dire, en fait, s'institutionnalisent, d'un mouvement inégal, la géographie, la sociologie et la statistique sociale. Problème, car institutionnalisation signifie autonomisation des méthodes et des savoirs et délimitation des frontières, alors que l'étude du phénomène urbain transgresse les partages de disciplines.

L'essai s'attache d'abord à suivre le mouvement d'institutionnalisation de la géographie et de la sociologie repérable à plusieurs caractères, comme le passage de l'amateurisme au professionnalisme, le recours à des principes spécifiques d'explication et à des méthodes privilégiées ou particulières, l'organisation d'écoles ou plutôt (terme que préfère l'auteur) d' " environnements " intellectuels où se reconnaissent les adhérents des causes en présence. Vidal de La Blache lance les Annales de géographie et se prononce pour un déterminisme spatial tempéré par l'action humaine dans un accord (l'« harmonisme », dit G. Montigny) que l'instrument privilégié des monographies régionales donne le mieux à voir. Durkheim lance L'année sociologique et préconise l'établissement de vastes synthèses à l'échelle de l'humanité qui font ressortir les lois générales du fonctionnement social.

Cette différence de présupposés et d'objectifs explique que l'intérêt des géographes vidaliens pour les études urbaines ait été plus tardif que celui des sociologues durkheimiens. Mais l'attrait de l'essai n'est pas seulement de marquer ces grandes oppositions. Il est aussi et surtout de ressusciter minutieusement les œuvres marquantes, et parfois ignorées, des géographes et des sociologues en les présentant et en les discutant. Du côté des géographes : Désiré Pasquet, connu pour son ouvrage sur Londres et les ouvriers de Londres, Ch. Anthelme Roux, auteur d'une étude pionnière du Cours Berriat à Grenoble, Jacques Levainville, industriel devenu géographe de Rouen. Du côté des sociologues : l'œuvre, considérable de $\mathbf{M}$. Halbwachs, bien entendu, mais aussi le brillant travail de René Maunier l'inclassable sur L'Origine et la fonction économique des villes, etc.

La dernière partie de l'essai est consacrée à l'apport des statisticiens sociaux, tout particulièrement mal connu. Parmi eux : Émile Levasseur, sommité scientifique de l'époque et précurseur oublié de la géographie quantitative, et Paul Meuriot, modeste professeur de l'enseignement secondaire et auteur d'une ceuvre imposante consacrée notamment aux problèmes des centres-villes et d'agglomération urbaine - sans oublier Legoyt, Foville, etc.

Remarquable outil de travail, cet essai est destiné à devenir un ouvrage de référence pour tous les spécialistes de l'histoire des recherches urbaines.

Michel Амıт. 